\title{
Numerical Simulation and Modeling of
}

\section{UNSA91060 for Heat Transfer in Four-Stroke ICE Cylinder Head}

\author{
Olaiya Kamorudeen Adesina ${ }^{1}$, Alabi Ismaila Olanrewaju ${ }^{2}$, Falana Kafayat Abolanle ${ }^{3}$
}

${ }^{1}$ Department of Mechatronics Engineering, The Polytechnic, Ibadan, Nigeria

${ }^{2,3}$ Department of Mechanical Engineering, The Polytechnic, Ibadan, Nigeria

\begin{abstract}
Heat transfer is one of a number of indispensable tools in studying of ICEs, due to its influence on decisive parameters of operation like temperature and pressure inside the cylinder. It is safe to say that modeling of the engine heat transfer is among the most complex problems for engineers. Application of numerical methods to predict the heat transfer in a cylinder of reciprocating ICEs is a process of high importance, which was recognized from the earliest stages of their development. This is done to examine performance optimization and design improvement in order to meet nowadays demands exhibited on the engines. This present study focuses on a 3-D transient state temperature distribution analysis on a gasoline engine model via formulated of models, simulating using FEM in-built in the COMSOL Multiphysics software 4.3 a to determine the temperature distribution and gradient of the engine cylinder head model. The number of degrees of freedom solved for were 32685 in 383 s (12 minutes, 20 seconds) in the mesh optimization. From the result obtained it was discovered that the heat transfer in the combustion chamber of the ICE varies with time. Thus, it took the engine 10 minutes to complete a cycle vis-a-vis transfer of heat after combustion and that the heat transfer starts after 30 seconds of combustion. In addition, the temperature of the cylinder dropped from $1273.2 \mathrm{~K}$ to $301 \mathrm{~K}$ over a period of 10 minute.
\end{abstract}

Keywords - Comsol, Heat transfer, ICE, Simulation, $1 D$ model.

\section{INTRODUCTION}

One of the greatest contributors to engine energy losses is heat loss. Almost one third of the heat released during combustion is lost to cooling media and exhaust gases, thus dominating the Internal Combustion Engine (ICE) energy balance (Dahlstrom, 2016). The heat transfer from combustion gases to the coolant in reciprocating ICEs varies between $25-35 \%$ of the total energy released by the mixture of fuel and combustion air. Nearly half of the heat passes through the cylinder walls and most of the remaining heat is transferred to the coolant in the cylinder head with the most intensive heat transfer near the exhaust valve seats (Oleg, 2013).

During engine operation, there are energy losses via heat transfer to the cylinder walls during the latter stages of the compression stroke and the combustion-expansion stroke. During the compression process, the charge temperature rises above the wall temperature and thus heat transfer occurs from the in-cylinder gases to the cylinder walls. In the combustion-expansion stroke, the heat transfer rate to the cylinder walls is the highest (Wei et al., 2016).
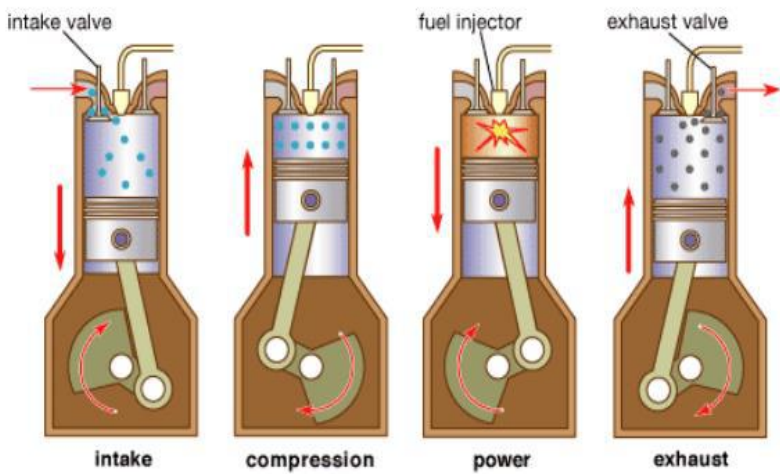

Fig. 1: A four-stroke ignition cycle (António, 2008)

Modeling of the heat transfer is usually considered as a part of whole engine simulation including combustion modeling, emission and soot formation modeling, fuel spray modeling etc., and can serve as a prerequisite of performance optimization and design improvement in order to meet nowadays demands added on the engines. There are two basic types of models that are in use for modeling of the heat transfer: thermodynamic and multidimensional models. The simplest type of thermodynamic models are zero dimensional models or global models, which suits for estimating of the general parameters by use of semi empiric correlations. Another kind of thermodynamic models is quasi dimensional models. Currently, researchers tend to move towards more comprehensive and accurate models describing the 
performance of the engine, although, in some cases proved to be impractical for their complexity. Multidimensional models are increasingly gaining more attention due to their ability to compute the gas motion by numerical solving of the differential equations representing the laws of mass, energy and momentum conservation, thus, providing a greater deal of precision (Oleg, 2013).

Global approach was employed by Ziarati (1990) in 1996 to study the delay period and droplet penetration. Thanks to the developed model which used the Annand's correlation to describe the heat transfer in the combustion chamber and was tested against the experimental results of a direct injection diesel engine (Ricardo, Atlas) for a number of nozzle configuration, plunger size and engine speeds and loads. Zarati managed to obtain good agreement of cylinder pressure and temperature with experimental results

However, many attempts were made to study and understand the causes of these heat losses and what amendments could be done, mainly through simulations and experimental work (Dahlstrom, 2016). According to Vijayakumaran et al. (2014), any physical fluid flow problem can be solved either experimentally or numerically. In the recent years there were tremendous improvement in the field of numerical techniques which made a great impact on the evaluation of complex flow problems and achieving their solution.

$\mathrm{Xu}$ and Cho (2016) reported that In-cylinder flow simulation experiment works have been carried out using a hot wire velocimetry or laser Doppler velocimetry (LDV) to measure the velocity field. This test is very difficult because the characteristics measured in the reciprocating engine cylinder flow are a highly complex three-dimensional turbulence and instability. The paper therefore concluded that the numerical approach could be changed by developing a CFD model for the in-cylinder flow predictions.

Kurniawan and Abdullah (2008) performed the CFD simulation for the combustion process in their analysis using the moving mesh and boundary algorithm where every event represents the different mesh and boundary geometries for every different crank angle in each step of engine cycle. Thus, in order to perform CFD simulation for internal combustion process, the analysis was carried out by using transient, moving meshes and boundaries, high compressible Reynolds number, high turbulence intensity, momentum, heat and mass transfer with complex geometries model and chemical-thermal dependent as well.

As reported by Meman et al. (2016), Cheng x. et al. (2015) analyzed on the cylinder head of the heavy diesel engine by performing 3-dimensional CFD analysis with the cylinder combustion and an engine cooling jacket, www.ijaems.com stress and strain analysis under thermal-mechanical coupling conditions and high cycle fatigue analysis and also they had measured temperature distribution on the engine head and predicted the danger region through calculation matches the crack in actual experiment. New thermo-mechanical analysis of cylinder head was investigated by Fadaei et al. (2011) in this paper the 3D model was created by using Solid Works 2008. The mesh and analysis of cylinder head were constructed in ANSYS.

Meman et al. (2016) also reported the work of Baox in Zhao et al. (2012) and the paper evaluated the problem of super heat generation in cylinder head. The simulation of heat transfer was taken out for the combustion chamber by help of the coupled finite element method (FEM). Hence, the temperature distribution at cylinder blocks, cylinder, cylinder head, cylinder gasket, valve seat and valve guide were measured.

Oke et al. (2015) confronted the problem of heat transfer in spark ignition engine by developing a model for 2 stroke spark ignition engine and used Comsol Multiphysics to predict the temperature distributions and heat transfer on the combustion chamber.

Despite all these studies regarding the effect of heat transfer on the combustion chamber walls of Internal Combustion Engines (ICE), there are still areas which have yet to be examined. This work concerns heat transfer principles in four strokes ICE from modeling and simulation standpoint.

\section{METHODOLOGY}

\subsection{Modeling and Simulation}

\subsubsection{Model Formulation - One Dimensional Solution to the Problem \\ Domain Equation}

Starting with the time dependent heat conduction equation

$$
\rho c_{p} \frac{\partial T}{\partial t}+\nabla \cdot(-k \nabla T)=Q
$$

in Cartesian form

$$
\rho c_{p} \frac{\partial T}{\partial t}+\frac{\partial}{\partial \mathrm{x}}\left(-k_{x} \frac{\partial \mathrm{T}}{\partial \mathrm{x}}\right)=Q
$$

To avoid division by zero at $\mathrm{x}=0$, which creates numerical problems, multiply equation (2) by $\mathrm{x}^{2}$

$$
x^{2} \rho c_{p} \frac{\partial T}{\partial t}+\frac{\partial}{\partial \mathrm{x}}\left(-k_{x} x^{2} \frac{\partial \mathrm{T}}{\partial \mathrm{x}}\right)=x^{2} Q
$$

Using dimensionless coordinate $x$ by scaling the equation provides the option to quickly change the wall thickness without changing or parameterizing the geometry size. Introducing the dimensionless coordinate,

$$
\hat{x}=\frac{x}{w_{x}}, \frac{\partial}{\partial x}=\frac{1}{w_{x}} \frac{\partial}{\partial \hat{x}}
$$

and substituting in equation (iii) leads to

$$
\hat{x}^{2} w_{x}^{2} \rho c_{p} \frac{\partial T}{\partial t}+\frac{1}{w_{x}} \frac{\partial}{\partial \hat{\mathrm{x}}}\left(-k_{x} \hat{x}^{2} w_{x}^{2} \frac{\partial \mathrm{T}}{\partial \hat{\mathrm{x}}}\right)=\hat{x}^{2} w_{x}^{2} Q
$$

Page | 136 


$$
\hat{x}^{2} \rho c_{p} \frac{\partial T}{\partial t}+\frac{\partial}{\partial \hat{x}}\left(-\frac{k_{x} \hat{x}^{2}}{w_{x}} \frac{\partial T}{\partial \grave{x}}\right)=\hat{x}^{2} Q \quad \text { (iv) }
$$

On the domain

$$
\mathbf{x}=\mathbf{0} \quad \mathbf{x}=\mathbf{1}
$$

\section{Comsol implementation}

To implement equation (iv) and the boundary conditions of this problem, an 1D time-dependent equation version of the General Form PDE interface given in equation (v) was used:

$$
\begin{aligned}
& e_{a} \frac{\partial^{2} u}{\partial t^{2}}+d_{a} \frac{\partial u}{\partial t}+\nabla \cdot \Gamma=F \\
& {\left[\begin{array}{ll}
e_{a} \frac{\partial^{2} u}{\partial t^{2}}+d_{a} \frac{\partial u}{\partial t}+\nabla . \Gamma=F & \text { in } \Omega \\
-n \Gamma=G+\left(\frac{\partial w_{x}}{\partial u}\right)^{T} \mu & \text { on } \partial \Omega(\mathrm{v}) \\
0=w_{x} & \text { on } \partial \Omega
\end{array}\right.}
\end{aligned}
$$

Comparing equation (iv) to

$$
\begin{gathered}
\boldsymbol{e}_{\boldsymbol{a}} \frac{\boldsymbol{\partial}^{2} \boldsymbol{u}}{\partial \boldsymbol{t}^{2}}+\boldsymbol{d}_{\boldsymbol{a}} \frac{\partial \boldsymbol{u}}{\partial \boldsymbol{t}}+\nabla \cdot \Gamma=\boldsymbol{F} \\
\boldsymbol{e}_{\boldsymbol{a}}=\mathbf{0} \\
\boldsymbol{d}_{\boldsymbol{a}}=\widehat{\boldsymbol{x}}^{2} \boldsymbol{\rho} \boldsymbol{c}_{\boldsymbol{p}} \\
\Gamma=-\frac{k_{x} \hat{x}^{2}}{w_{x}} \frac{\partial \mathrm{T}}{\partial \hat{\mathrm{x}}} \\
Q=0
\end{gathered}
$$

\section{Boundary and initial conditions}

Because of symmetry about $x=0$, there is no flux through this point meaning

$$
\frac{\partial T}{\partial \hat{x}}=0
$$

At the surface $\mathrm{x}=1$, a convective heating and cooling expression with a heat transfer coefficient $h_{\mathrm{s}}\left[\mathrm{W} /\left(\mathrm{m}^{2} \cdot \mathrm{K}\right)\right]$, for the influx of heat $\left(\mathrm{W} / \mathrm{m}^{2}\right)$.

Since the flux,

$$
q_{\text {in }}=h_{s}\left(T_{\text {ext }}-T\right)
$$

$$
\Gamma=-\frac{k_{x} \hat{x}^{2}}{w_{x}} \frac{\partial \mathrm{T}}{\partial \hat{\mathrm{x}}}
$$

and

$$
-k_{x} \frac{\partial \mathrm{T}}{\partial \hat{\mathrm{x}}}=h_{s}\left(T_{e x t}-T\right)
$$

Therefore,

$$
g=-\frac{\hat{x}^{2}}{w_{x}} h_{s}\left(T_{e x t}-T\right)
$$

\subsubsection{COMSOL Multiphysics 4.3a}

COMSOL Multiphysics 4.3a software package which is a powerful finite element method (FEM) and partial differential equation (PDE) solution engine was used for the analysis. It has ten (10) add-on modules that expand the capabilities in the following application areas (Alabi et al., 2015): AC/DC, Acoustics, Chemical Species and Transport, Electrochemistry, Fluid flow, Heat transfer, Plasma, Radio Frequency (RF), Structural Mechanics, and Mathematics Modules.
Fig. 2 below is the schematic representation of Comsol Multiphysics processes or steps as applicable to this work.

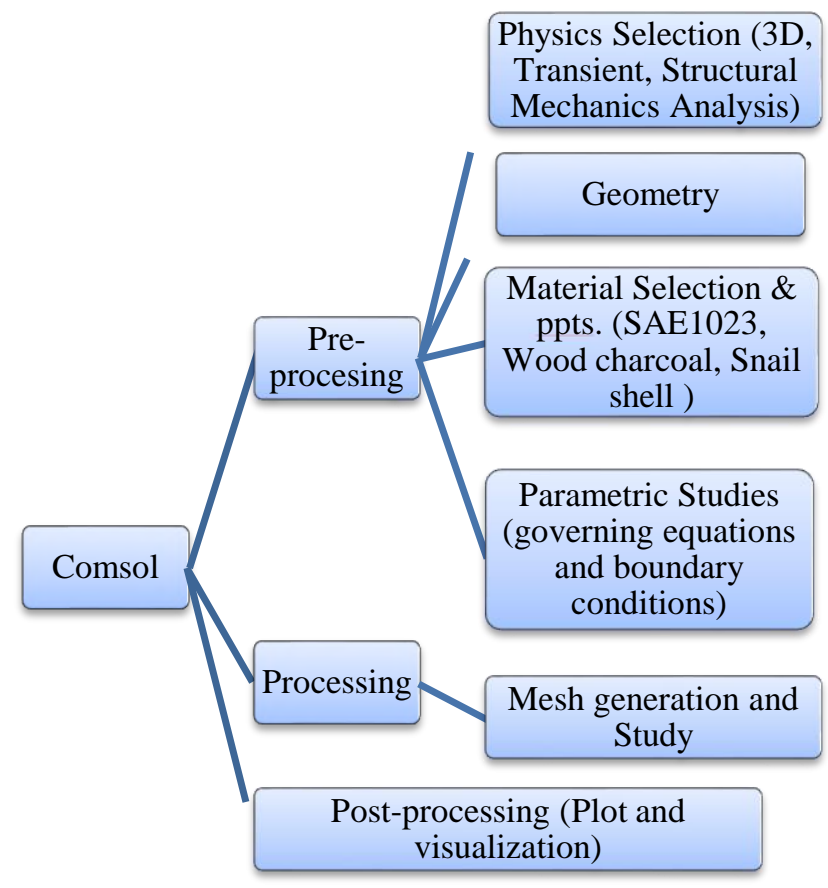

Fig. 2: Comsol Schematic Representation of the Study

\section{Model Pre-processing}

The Structural Mechanics add-on module which has a Thermal-Strength in-built model was used for the simulation and analysis.

$$
\rho c_{p} \frac{\partial T}{\partial t}=\nabla \cdot(k \nabla T)
$$

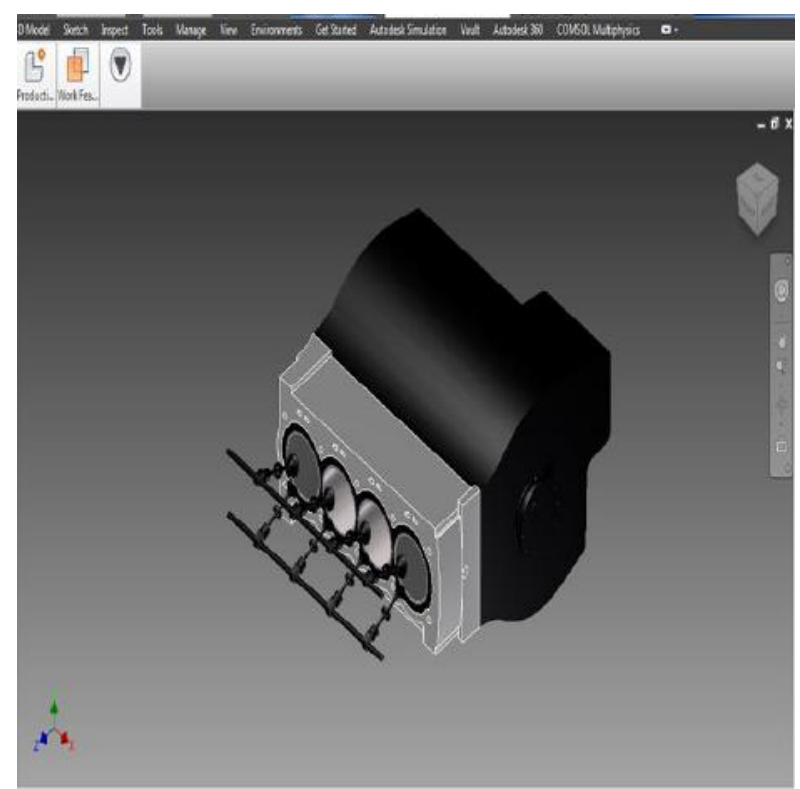

Fig.3: Geometry model window in Inventor 


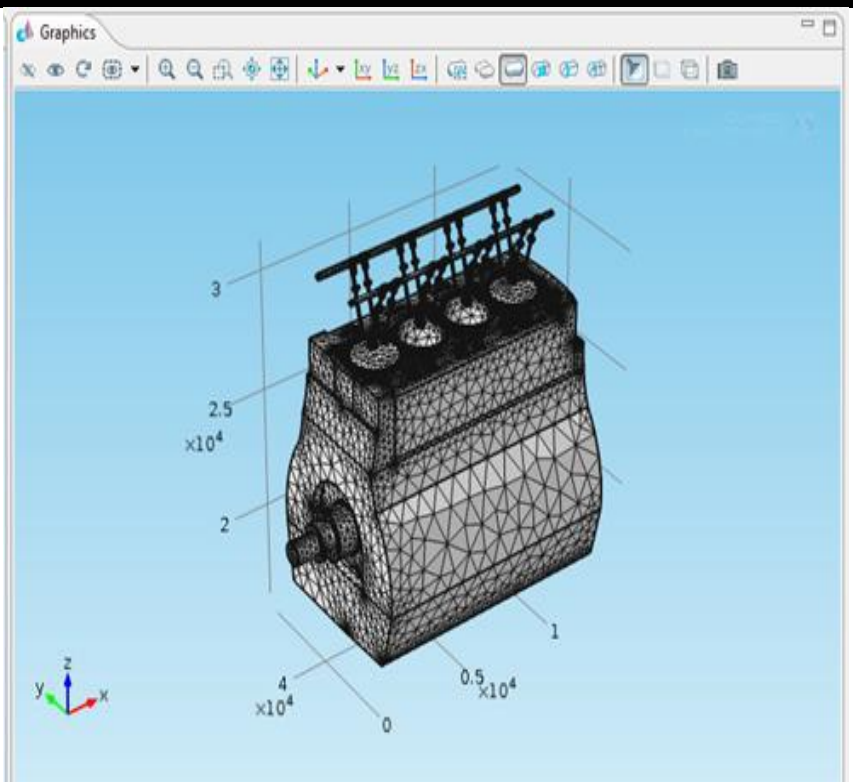

Fig. 4: Mesh representation of the study

\section{Model-Processing}

Mesh Generation: The mesh chosen was Free Tetrahedral. The Completed mesh consists of larger number of elements and degrees of freedoms in the best possible period.

\section{Model-Post-processing and Visualization}

In post processing mode different plots types were obtained from sets of selected parameters. The solution variables were later visualized using the post processing utilities for their derivative, and the space coordinates. Many frequently used expressions were predefined as application mode variables, directly available from lists in the Plot Parameters.

\section{RESULTS AND DISCUSSION}

The results are presented as shown in the figures below.

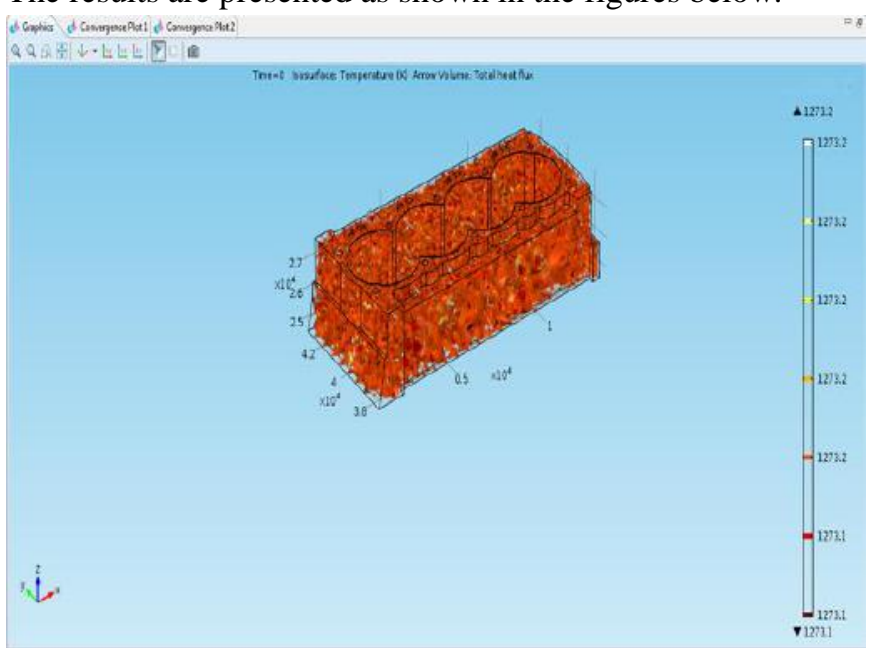

Fig. 5: Full-cylinder head Model at initial combustion

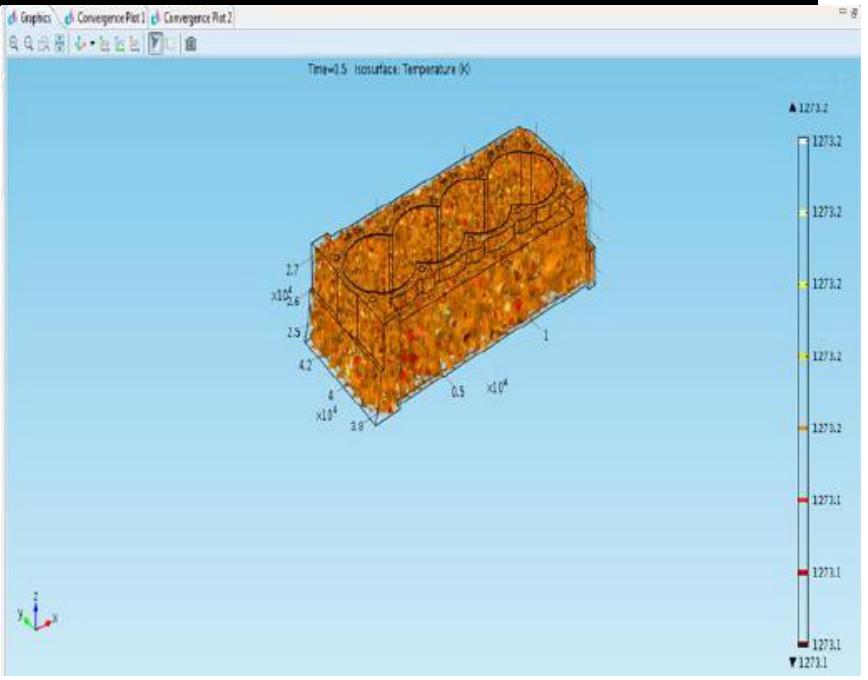

Fig. 6: Model after 30 seconds of combustion

Fig. 5 shows the full cylinder model. The cylinder reached its maximum temperature of $1273.2 \mathrm{~K}$ at the earliest stage of combustion which is when the combustion just occurs. After 0.5 minutes heat transfer process began and traces of red hot start disappearing and this could be seen in fig. 6 .

From fig. 7 more disappearance of heat was pronounced and After 9 minutes the traces of heat as shown in fig. 8 has completely disappear. Fig. 9 shows the time dependent solver while fig. 10 is the result obtained from the 1-D heat transfer model, which is the plot of temperature against time. It was observed that the temperature of the cylinder dropped from $1273.2 \mathrm{~K}$ to 301 $\mathrm{K}$ over a period of 10 minute. This is an indication that a complete cycle took 10 minute to transfer heat after combustion, and that the heat transfer starts after 30 seconds of combustion.

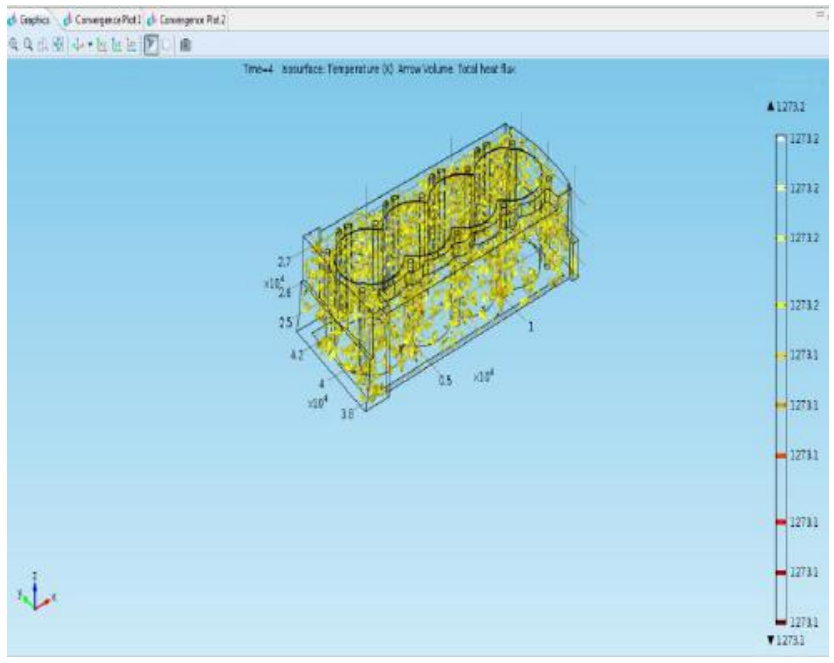

Fig. 7: Heat Transfer after 4 minutes 


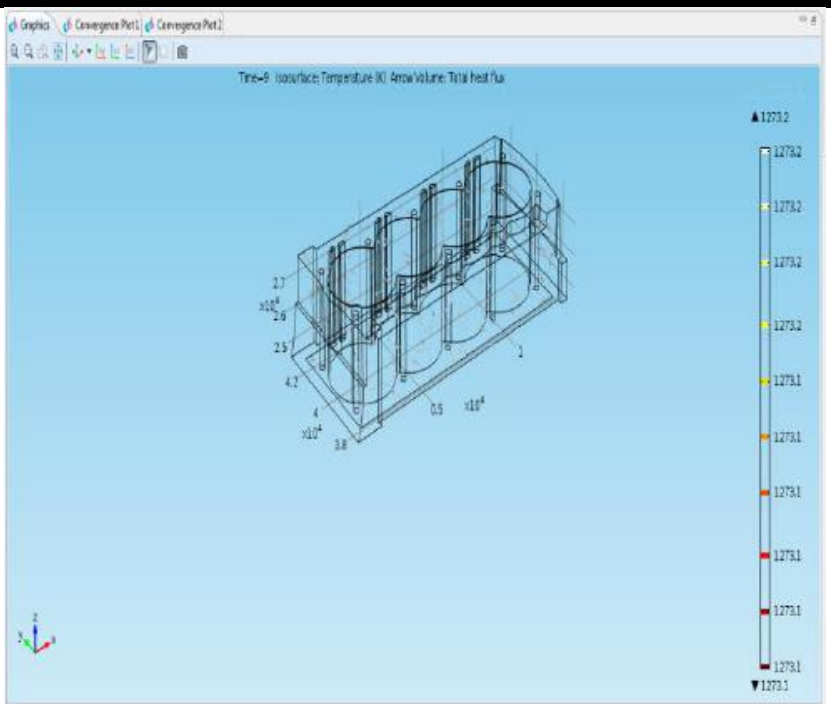

Fig. 8: Heat transferred completely in 9 minutes

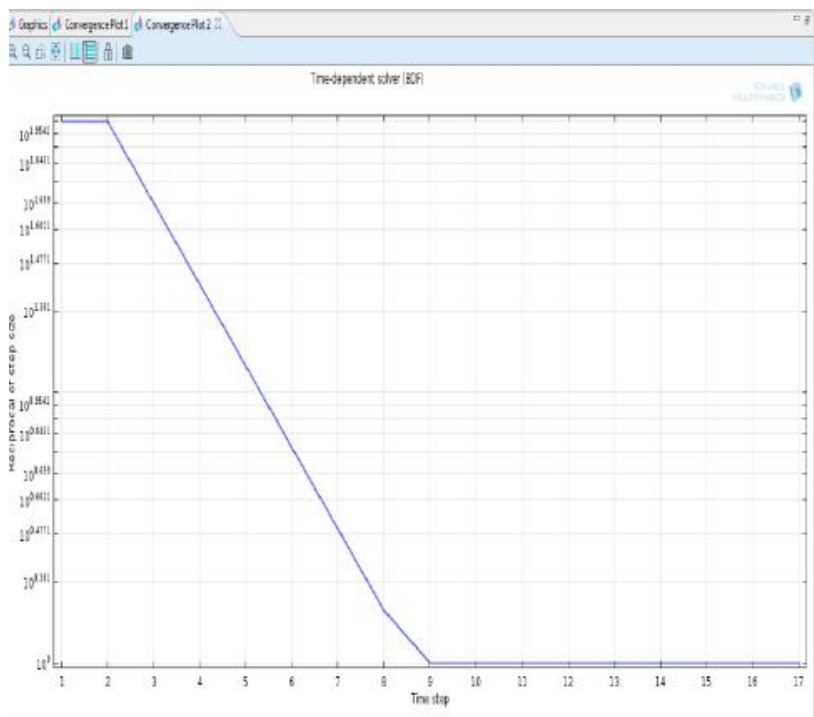

Fig. 9: Time Dependent Solver

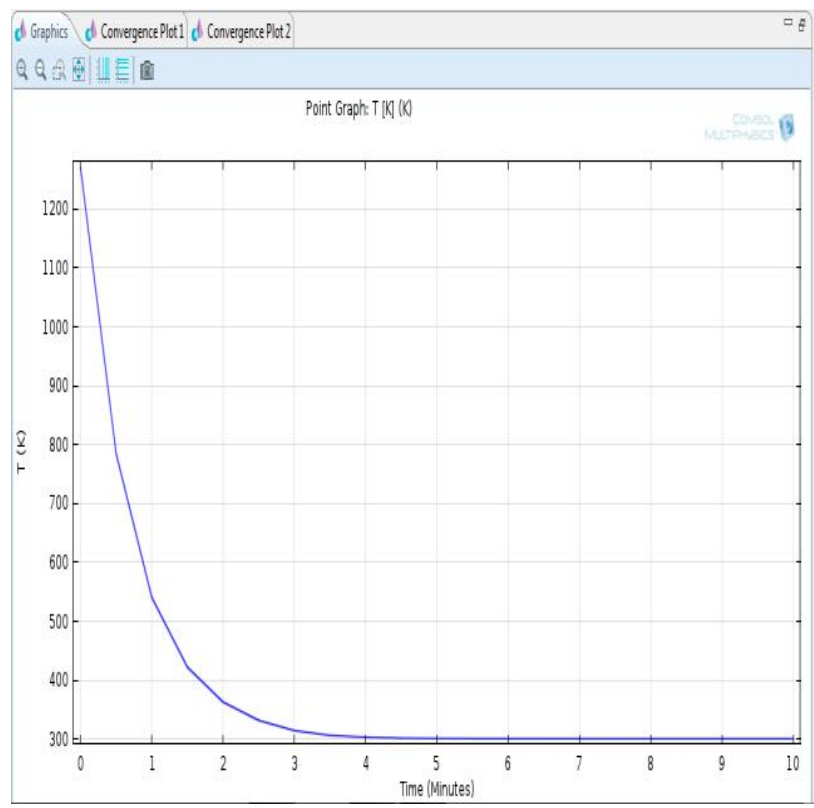

Fig. 10: Plot of temperature against time

\section{CONCLUSION}

The energy losses resulting from the transfer of heat to the cylinder walls is mainly through the piston crown, cylinder liner, combustion chamber, and valves. A decrease in the surface temperature of these components leads to a reduction of the transfer of heat to the engine coolant and thus reduces energy losses (Wei et al., 2016). Thus, the heat transfer in the combustion chamber of the ICE varies with respect to time. The obtained results showed that it took the engine 10 minutes to complete a cycle vis-a-vis transfer of heat after combustion, and that the heat transfer starts after 30 seconds of combustion. In addition, the temperature of the cylinder dropped from $1273.2 \mathrm{~K}$ to $301 \mathrm{~K}$ over a period of 10 minute.

An advancement in technology leads to this research, for improvement and longevity in four stroke engine performance. The results obtained can be validated using other available software packages like ANSYS, ABAQUS, NASTRAN, MATLAB, J-ANALYZER used in simulating the heat transfer on IC Engines.

\section{REFERENCES}

[1] Alabi, I.O, Olaiya, K.A, Kareem, M.O. (2015) 'Numerical Simulation of Temperature Distribution in A Tri-Cycle Engine Piston. International Journal of Scientific \& Engineering Research, Volume 6, Issue 7, pp. 252-258

[2] Baoxin Z., Dingwei G., Jingqian S., Zheng Z., Hao G., Gang L., and Ying G., (2012) "Experiment and Numerical Analysis of Temperature Field of Cylinder Head Based on a GW4D20 Diesel Engine, Volume:190, pp. 965-976.

[3] Cheng X., Wang X., Ming Y., Hongfei Z., and Ran G. (2015) Thermal-Mechanical Fatigue Analysis of Diesel Engine Cylinder Head Based on FluidStructure Interaction.”, SAE Technical Paper 201501-0558, doi:10.4271/2015-01-0558.

[4] Dahlstrom J (2016). Experimental Investigations of Combustion Chamber Heat Transfer in a Light-Duty Diesel Engine, Thesis for the degree of Doctor of Technology, Faculty of Engineering of Lund University,pp. 1-123.

[5] Fadaei M., Vafadar H. and Noorpoor A. (2011) New Thermao-Mechanical Analysis of Cylinder Heads Multi-Field Approach.”, Open Access Funded by Sharif University of Technology, Scientia Iranica, Volume:18, Issue:1, pp:66-74.

[6] Kurniawan W.H. and Abdullah S. (2008) Numerical Analysis of the Combustion Process in A FourStroke Compressed Natural Gas Engine with Direct Injection System. J. Mech. Sci. Tech., 22, pp. 19371944.

[7] Meman M.J, Solanki A.B and Parmar A.J (2016), Design, Modeling and Analysis of Structural

Page | 139 
Strength of Cylinder and Cylinder Head of 4-stroke (10 H.P.) C.I. Engine - A Review, International Journal of Advanced Engineering, Management and Science, Vol-2, Issue-4, pp. 156-162.

[8] Oke D.B, Alabi I.O, and Adegbola A.A (2016) Heat Transfer Analysis in Internal Combustion Engine Piston Using Comsol Multiphysics: A Case Study of Tri-Cycle, International Journal of Scientific \& Engineering Research, Volume 7, Issue 7, pp. 59-64.

[9] Oleg S (2013). Heat Transfer Inside Internal Combustion Engine: Modelling and Comparison with Experimental Data, Master's thesis submitted to Faculty of Technology, Lappeenranta University of Technology, pp. 1-55.

[10] Vijayakumaran P., Elayaraja R., Muthuvel A., Subramanian M., Murukesan R. (2014) Numerical Simulation of Combution Chamber Geometry on A H.S.D.I. Diesel Engine - A CFD Approach, IOSR Journal of Mechanical and Civil Engineering,2320334X; pp. 66-73.

[11] Wei M, Nguyen T.S, Turkson R.F, Guo G and Liu J (2016). The Effect of Water Injection on the Control of In-Cylinder Pressure and Enhanced Power Output in a Four-Stroke Spark-Ignition Engine, Sustainability 2016, 8,993; pp. 1-22

[12] Xu C.C and and Cho H.M (2016) The Analysis of the CFD about the Swirl Generation in Four- Stroke Engine, International Journal of Applied Engineering Research ISSN 0973-4562 Volume 11, Number 16, pp. 8940-8945.

[13]Ziarati R. 1990. Mathematical modeling and computer simulation of medium size engine running on varying quality fuels. International symposium on COMO - DIA. 